\title{
PRÉSENTATION DE LA PROBLÉMATIQUE DU BIEN-ÊTRE DES ANIMAUX
}

\author{
INTRODUCTION TO ANIMAL WELFARE
}

Par Pierre MORMEDE ${ }^{1}$

(Communication présentée le 05 novembre 2020, manuscrit accepté le 15 février 2020)

Mots-clés : bien-être animal, bientraitance, sensibilité, conscience

Key words: animal welfare, sentience, well-being, consciousness

\section{INTRODUCTION}

Les relations des humains aux autres animaux est un thème récurrent qui a pris une importance croissante depuis quelques années dans le débat public. Que ce soit dans le contexte de l'élevage de production vivrière, de l'utilisation des animaux pour la recherche biologique et médicale, des animaux de compagnie, de la faune sauvage captive ou en liberté, notre rapport aux animaux est l'objet d'une attention renouvelée qui débouche souvent sur des remises en question de pratiques telles que l'enfermement des animaux sauvages dans les cirques et zoos, les conditions de l'élevage de production, les conditions de vie des animaux familiers, certaines pratiques de chasse de la faune sauvage. Ces interrogations se nourrissent des réflexions philosophiques qui questionnent le statut des humains dans le règne animal et les rapports moraux et juridiques entre les humains et les autres animaux (Jeangène Vilmer, 2018). Elles tiennent compte également de la progression des connaissances scientifiques sur les capacités psychiques des animaux (Le Neindre et al. 2009 ; 2017). La question du bien-être a cristallisé les démarches visant à fournir aux animaux des conditions de vie qui respectent leurs besoins éthologiques. Cette question est à la croisée de nombreuses influences parfois contradictoires, philosophiques et morales, scientifiques, technologiques, réglementaires et sociétales (Mormede et al. 2018). Les bases de cette réflexion ont été jetées il y a plus d'un demi-siècle (Hediger, 1950 ; 1955, pour les animaux sauvages en captivité ; Russell et Burch, 1959, pour l'utilisation des animaux en expérimentation; Rapport Brambell (HMSO, 1965), pour les animaux de rente), mais le réel intérêt des citoyens pour cette question est beaucoup plus récent (Commission européenne, 2016). Le bien-être des animaux a été progressivement pris en compte par les professionnels de l'élevage, du secteur agroalimentaire et de la distribution (BBFAW, 2019). C'est aujourd'hui un enjeu majeur pour l'évolution de l'élevage et il prend une place importante dans tous les plans de développement des filières animales, en écho à la stratégie des pouvoirs publics "Le bien-être animal au cour d'une activité durable " (https://agr iculture.gouv.fr/2016-2020-une-strategie-globale-pourle-bien-etre-des-animaux-en-france). L'un des accomplissements importants en a été la mise en place en 2017 du Centre national de référence pour le bien-être animal (www.cnr-bea.fr). Plus récemment encore, la question du bien-être des animaux s'impose dans l'agenda politique. Du fait de leur formation et de leur place sur le terrain, les vétérinaires doivent être au cour de cette relation humains-animaux, comme « experts garants du bien-être animal » (Ordre national des vétérinaires, 2015).

\section{ÉVOLUTION DU STATUT DES ANIMAUX}

Dans la tradition philosophique, la prise en compte du bien-être des animaux répond au souci moral dont ceux-ci doivent faire l'objet du fait de leur nature sensible. Ainsi, Jean-Jacques Rousseau (1755) déclarait : «Il semble, en effet, que si je suis obligé de ne faire aucun mal à mon semblable, c'est moins parce qu'il est un être raisonnable que parce qu'il est un être sensible, qualité qui étant commune à la bête et à l'homme, doit au moins donner à l'une le droit de n'être point maltraitée inutilement par l'autre ». La réflexion philosophique s'est principalement centrée sur l'objectif de limitation des souffrances. Jeremy Bentham (1789) par exemple déclarait au sujet des animaux : «La question n'est pas : Peuvent-ils raisonner? ou : Peuvent-ils parler? Mais : Peuvent-ils souffrir?». De fait, la première loi de 
protection animale fut la loi Grammont du 2 juillet 1850 qui incrimina les mauvais traitements exercés en public et abusivement contre les animaux domestiques. Il s'agissait cependant dans l'esprit des auteurs davantage de protéger la moralité publique que l'animal lui-même. Ultérieurement, c'est la nature sensible des animaux qui a été à la base de la règlementation qui a imposé des obligations positives au propriétaire quant à la manière de traiter ses animaux. C'est ainsi que la loi du 10 juillet 1976 sur la protection de la nature reconnait en son article 9 que « tout animal étant un être sensible doit être placé par son propriétaire dans des conditions compatibles avec les impératifs biologiques de son espèce ". Cet article, toujours en vigueur aujourd'hui, est codifié à l'article L214-1 du Code rural. Le bien-être des animaux est une valeur communautaire consacrée dans le Traité sur le fonctionnement de l'Union européenne (2012, Art. 13) : "Lorsqu'ils formulent et mettent en œuvre la politique de l'Union dans les domaines de l'agriculture, de la pêche, des transports, du marché intérieur, de la recherche et développement technologique et de l'espace, l'Union et les États membres tiennent pleinement compte des exigences du bien-être des animaux en tant qu'êtres sensibles... ». Une abondante réglementation élaborée au niveau du Conseil de l'Europe et de l'Union européenne et déclinée dans le droit national encadre désormais les activités d'élevage dans le respect du bien-être des animaux. La reconnaissance législative de la sensibilité des animaux a finalement été introduite dans le Code civil (Art. 515-14) par la loi du 16 février 2015 : « Les animaux sont des êtres vivants doués de sensibilité. Sous réserve des lois qui les protègent, les animaux sont soumis au régime des biens. " Cet article traduit l'évolution du droit vers une reconnaissance de la personnalité juridique des animaux.

\section{ANIMAUX D'ÉLEVAGE : LE RAPPORT BRAMBELL}

Le thème du bien-être des animaux en élevage a pris de l'importance dès les années 1960 avec le rapport Brambell (HMSO, 1965) en Grande-Bretagne, suite à la publication par Ruth Harrison (1964) d'un ouvrage très critique sur les conditions de l'élevage moderne. Ce rapport a proposé une définition du bien-être qui, avec le recul, apparaît très moderne, et a établi des normes minimales pour un bien-être acceptable en vue de satisfaire les besoins fondamentaux des animaux. L'énoncé de ces normes a été repris par le Farm animal welfare council (FAWC, 1979, 2009) et popularisé sous l'appellation des "Cinq libertés " (physiologique, environnementale, sanitaire, comportementale et mentale). Cette approche reste à la base des démarches pour la bientraitance (Milhaud, 2007) et le bien-être des animaux.

\section{UNE DÉFINITION DU BIEN-ÊTRE}

Le progrès des connaissances sur les capacités psychiques des animaux a conduit à leur reconnaître un certain niveau de conscience qui permet à l'animal en tant qu'individu de caractériser la situation dans laquelle il vit (Le Neindre et al. 2017). Il nous faut donc prendre en compte le ressenti des animaux dans l'évaluation de leur bien-être. Ce point de vue a été réaffirmé dans la définition du bien-être animal proposée par l'Anses en 2018 : « Le bien-être d'un animal est l'état mental et physique positif lié à la satisfaction de ses besoins physiologiques et comportementaux, ainsi que de ses attentes. Cet état varie en fonction de la perception de la situation par l'animal». La dimension mentale porte l'attention sur le fait qu'une bonne santé, un niveau de production satisfaisant ou une absence de stress ne suffisent pas. Il faut aussi se soucier de ce que l'animal ressent, des perceptions subjectives déplaisantes, telles que la douleur et la souffrance, mais aussi rechercher les signes d'expression d'émotions positives, satisfaction, plaisir... (Boissy et al. 2007).

\section{ÉVALUATION DU BIEN-ÊTRE}

L'évaluation de l'état de bien-être des animaux doit donc mettre en œuvre une approche intégrée combinant l'analyse des ressources et de l'état des animaux. Les ressources comprennent les caractéristiques de l'animal telles que la génétique ou son niveau de production, les propriétés de l'environnement physique et social, le mode de conduite et les rapports humains-animaux dans le contexte analysé. Elles permettent de s'assurer que les conditions du bien-être des animaux sont bien réunies (bientraitance). Ce sont également les leviers sur lesquels il sera possible d'agir dans une perspective d'amélioration (Whay, 2007). Cependant, c'est l'étude des comportements et de l'état physiologique et sanitaire de l'animal qui donne une vision intégrée de son adaptation à l'environnement et de son bien-être. De nombreux protocoles d'évaluation du bien-être ont été élaborés. Ils reposent sur le respect des " cinq libertés " et incluent des mesures individuelles réalisées sur l'animal et une évaluation des ressources. Pour les animaux de rente, cette démarche intégrée a été formalisée dans les protocoles élaborés dans le cadre de vastes projets européens et qui sont les référentiels de base, "Welfare Quality® » pour les porcs, les bovins et les volailles (Veissier, 2010) et AWIN ("Animal Welfare Indicators Project ") pour les petits ruminants, les équidés et les dindes. De nombreux protocoles, souvent plus facilement applicables sur le terrain et adaptés à différents types de productions, sont développés par les professionnels. On peut aussi mentionner l'existence de grilles d'évaluation spécifiques, comme le guide d'évaluation du bien-être des animaux à l'abattoir de l'« American Meat Institute "(Grandin, 2013). Bien que cette expression soit souvent entendue et utilisée, parler du "bien-être " animal en abattoir apparaît comme un oxymoron. Il semblerait préférable de ne pas populariser cette formule.

\section{UN SEUL BIEN-ÊTRE}

Les préoccupations relatives au bien-être des animaux, et en particulier des animaux de production, ne doivent pas être perçues comme des contraintes supplémentaires imposées de l'extérieur sur une activité économique déjà très difficile. Il apparaît de plus en plus que le bien-être des animaux rejoint celui des éleveurs (satisfaction au travail, reconnaissance sociale...) et la protection de l'environnement, ainsi que formulé dans le concept " un seul bien-être " qui s'intègre dans les réflexions actuelles de l'agroécologie pour l'évolution de l'élevage (Coste et al. 2018). Il est important de partager les concepts et les approches de terrain pour en tenir pleinement compte. 


\section{RÉFÉRENCES}

-Anses. Avis de l'Anses relatif au "Bien-être animal : contexte, définition et évaluation ", 2018. Disponible à : https://www.anses.fr/fr/system/files/SABA2016SA0288.pdf (consulté le 02/12/2020).

- BBFAW. The Business Benchmark on Farm Animal Welfare, 2019. Disponible à : https://www.bbfaw.com/media/1793 $\angle$ bbfaw report 2019-v2.pdf (consulté le 02/12/2020).

- Bentham J, 1789. An Introduction to the principles of morals and legislation. Oxford, UK, Clarendon Press, 1907 reprint of 1823 Edition, 1, p 311.

- Boissy A, Manteuffel G, Jensen MB, Moe RO, Spruijt B, Keeling LJ et al. Assessment of positive emotions in animals to improve their welfare. Physiol Behav. 2007;92:375-397.

- Commission européenne. Eurobaromètre spécial 442. Attitudes des Européens à l'égard du bien-être animal. 2016. Disponible à : https://ec.europa.eu $\angle$ commfrontoffice/publicopinion/ index.cfm/ResultDoc/download/Doc umentKy/71653 (consulté le 02/12/2020)

- Coste C, Oggero N, Mormede P, Boissy A. Bien-être animal : Définition. Dictionnaire d'Agroécologie, 2018. Disponible à : https://dicoagroecologie.fr/encyclop edie/bien-etre-animal/ (consulté le 02/12/2020).

- FAWC. Farm Animal Welfare Council. Press statement, 1979. Disponible à: http://webarchive.nationalarchives gov.uk/20121007104210/http:/www fawc.org.uk/pdf/fivefreedoms1979.pdf (consulté le 02/12/2020).

- FAWC. FAWC Report on farm animal welfare in Great Britain: Past, present and future, 2009. Disponible à : https://www.gov.uk/government/publ ications/fawc-report-on-farm-animal-we lfare-in-great-britain-past-present-andfuture (consulté le 02/12/2020).

- Grandin T. Recommended animal handling, guidelines \& audit guide: a systematic approach to animal welfare. Washington DC, USA, American Meat Institute, 122 p, 2013. Disponible à : http://www.animalhandling.org Lsites/default/files/forms/animal-han dling-guidelines-Nov32017.pdf

http://grandin.com/interpreting.am i.guidelines.html (consultés le 02.12.2020).

- Harrison R. Animal Machines: The new factory farming industry. London, England, Vincent Stuart Publishers Limited, 186 pp,1964.

- Hediger H. Wild animals in captivity. London, England: Butterworth Scientific Publications, 1950.

- Hediger H. Studies of the psychology and behaviour of captive animals in zoos and circuses. London, England: Butterworths Scientific Publications, 1955.

-HMSO. Report of the technical committee to enquire into the welfare of animals kept under intensive livestock husbandry systems, Cmd. 2386 (Great Britain. Parliament), H.M. Stationery Office; 1965 [" Le Rapport Brambell ] http://docplayer.net/1260087-Techn ical-committee-to-enquire-into-the-welfare-of-animals-kept-under.html (consulté le 02/12/2020).

- Jeangène Vilmer JB. L'éthique animale. Paris, Presses Universitaires de France, Collection 'Que sais-je' $n^{\circ} 3902$; $3^{\circ}$ édition, 2018.

- Le Neindre P, Guatteo R, Guémené D, Guichet JL, Latouche K, Leterrier C et al. Douleurs animales. Les identifier, les comprendre, les limiter chez les animaux d'élevage. Expertise scientifique collective, rapport d'expertise. INRA, France. 2009. Disponible à : https://www6.paris.inra.fr/depe/M edia/Fichier/Expertises/Douleurs-an imales/Synthese-Douleurs-Animales ; https://www6.paris.inra.fr/depe/M edia/Fichier/Expertises/Douleurs-an imales/Rapport-complet-Douleurs-an imales (consultés le 02.12.2020)

- Le Neindre P, Bernard E, Boissy A, Boivin X, Calendreau L, Delon $\mathrm{N}$ et al. Animal consciousness. EFSA Supporting publication 2017:EN-1196. Disponible à : http://onlinelibrary.w iley.com/doi/10.2903/sp.e fsa.2017.EN-1196/epdf (consulté le 02.12.2020)

- Milhaud C. Rapport sur l'utilisation du néologisme "bientraitance " à propos de la protection des animaux. Académie vétérinaire de France, 2007. https://academie-veterinaire-d efrance.org/fileadmin/user upload/P ublication/PrisesPosition/AVF 2007. Bientraitance Rapport.pdf (consulté le 02/12/2020)

- Mormede P, Boisseau-Sowinski L, Chiron J, Diederich C, Eddison J, Guichet JL et al. Bien-être animal : contexte, définition, évaluation. INRA Productions Animales, 2018 ; 31:145-162. Disponible à : https://pr oductions-animales.org/article/view/2299 (consulté le 02/12/2020).

Ordre national des vétérinaires. 2015. https://static.veterinaire.fr/filea dmin/user upload/Actes colloque du 24 novembre 2015 CNOV.pdf

- Rousseau JJ. Discours sur l'origine et les fondements de l'inégalité parmi les hommes. 1755. Paris, France, Gallimard. La Pléiade, t. III, Préface, p126.

- Russell WMS, Burch RL. The principles of humane experimental technique. London GB, Methuen. 1959.

- Veissier I, Botreau R, Perny P. Évaluation multicritère appliquée au bien-être des animaux en ferme ou à l'abattoir : difficultés et solutions du projet

- Welfare Quality®. INRA Prod Anim. 2010;23:269-284.

- Whay HR. The journey to animal welfare improvement. Animal Welfare. 2007;16:117-122. 